\title{
SUBNORMAL OPERATORS QUASISIMILAR TO AN ISOMETRY ${ }^{1}$
}

BY

\author{
WILLIAM W. HASTINGS
}

\begin{abstract}
Let $V=V_{0} \oplus V_{1}$ be an isometry, where $V_{0}$ is unitary and $V_{1}$ is a unilateral shift of finite multiplicity $n$. Let $S=S_{0} \oplus S_{1}$ be a subnormal operator where $S_{0} \oplus S_{1}$ is the normal decomposition of $S$ into a normal operator $S_{0}$ and a completely nonnormal operator $S_{1}$. It is shown that $S$ is quasisimilar to $V$ if and only if $S_{0}$ is unitarily equivalent to $V_{0}$ and $S_{1}$ is quasisimilar to $V_{1}$. To prove this, a standard representation is developed for n-cyclic subnormal operators. Using this representation, the class of subnormal operators which are quasisimilar to $V_{1}$ is completely characterized.
\end{abstract}

0. Introduction. The purpose of this paper is to study quasisimilarity within the class of subnormal operators. Two Hilbert space operators $A$ and $B$ are quasisimilar if there exist operators $X$ and $Y$ which are one-to-one, have dense range, and satisfy $X A=B X$ and $A Y=Y B$. Quasisimilarity was introduced by Sz.-Nagy and Foiaş, who gave a simple characterization of the class of operators which are quasisimilar to a unitary operator [13]. They also showed that if $A$ is quasisimilar to a unitary operator $W$, then there is a one-to-one correspondence between the hyperinvariant subspaces of $A$ and the hyperinvariant subspaces of $W$. In general, it is known that if $A$ and $B$ are quasisimilar and $A$ has a hyperinvariant subspace, then so does $B$ [7].

An operator $S$ is subnormal if $S$ has an extension $T$ to a larger Hilbert space on which $T$ is normal. Let $\mu$ be a positive, finite Borel measure with compact support in $\mathbf{C}$ and let $U_{\mu}$ be the operation of multiplication by $z$ on $H^{2}(\mu)$, the closure in $L^{2}(\mu)$ of the polynomials in $z$. Up to unitary equivalence, the operator $U_{\mu}$ is the most general cyclic subnormal operator. If $m$ is Lebesgue measure on $\mathcal{T}=\{z:|z|=1\}$, then $H^{2}=H^{2}(m)$ is the classical Hardy space and $U_{m}$ is the unilateral shift. A measure $\mu$ is of type $\delta$ if

(1) $\mu$ is carried by $\{z:|z| \leqslant 1\}$,

(2) the restriction of $\mu$ to $\mathcal{T}$ is absolutely continuous,

Received by the editors April 22, 1976 and, in revised form, December 21, 1976 and October 4, 1978.

AMS (MOS) subject classifications (1970). Primary 47B20.

Key words and phrases. Subnormal operator, isometry, quasisimilar, normal decomposition, unilateral shift, dominant operator.

${ }^{1}$ The paper is part of the author's dissertation submitted in partial fulfillment of the requirements of the Ph. D. at The University of Michigan. The author gratefully acknowledges his indebtedness to Professor Allen Shields. 
(3) $\int \log (d \mu / d m) d m>-\infty$.

Recently, S. Clary proved that $U_{\mu} \sim \sim U_{m}$ if, and only if, $\mu$ is of type $\mathcal{S}$ [3], [4]. In this paper we will extend his result by replacing $U_{m}$ by more general isometric operators.

Each Hilbert space operator $A$ has an orthogonal decomposition $A=A_{0} \oplus$ $A_{1}$, where $A_{0}$ is normal and $A_{1}$ is completely nonnormal. By completely nonnormal we mean that the restriction of $A_{1}$ to a reducing subspace is never normal. Let $S$ and $S^{\prime}$ be subnormal operators with decompositions $S_{0} \oplus S_{1}$ and $S_{0}^{\prime} \oplus S_{1}^{\prime}$, respectively, and suppose $S$ and $S^{\prime}$ are quasisimilar. We will show that $S_{0}$ and $S_{0}^{\prime}$ must be unitarily equivalent. It is not necessary, however, that $S_{1}$ and $S_{1}^{\prime}$ be quasisimilar even if $S^{\prime}$ is an isometry. On the other hand, if $S_{1}^{\prime}$ is the unilateral shift of finite multiplicity $n$, then we will conclude that $S_{1}$ and $S_{1}^{\prime}$ are quasisimilar.

This brings us to the question of which subnormal operators are quasisimilar to the shift of multiplicity $n$. Such an operator must be $n$-cyclic. An operator $\boldsymbol{A}$ on a Hilbert space $\boldsymbol{H}$ is $n$-cyclic, or cyclic of finite order $n$, if there is a set of $n$ vectors $\left\{x_{1}, \ldots, x_{n}\right\} \subset H$ such that the smallest subspace of $H$ invariant under $A$ and containing $\left\{x_{1}, \ldots, x_{n}\right\}$ is all of $H$, while no set of $n-1$ vectors has this property. An $n$-cyclic subnormal operator has an $n^{\prime}$-cyclic normal extension where $n^{\prime}<n$. Using this fact, a standard representation is developed for $n$-cyclic subnormal operators. This model allows us to characterize the class of subnormal operators which are quasisimilar to the shift of multiplicity $n$.

1. Notation and definitions. All operators considered here are bounded Hilbert space operators. An operator $X$ is quasiinvertible if $X$ has dense range and no kernel. If two operators $A$ and $B$ are quasisimilar, we will write $A \sim \sim B$ or $A \sim \sim B$ via $(X, Y)$, where $X$ and $Y$ are quasiinvertible operators such that $X A=B X$ and $A Y=Y B$. If $A$ and $B$ are unitarily equivalent, then we will write $A \cong B$ or $A \cong B$ via $X$, where $X$ is unitary and $X A=B X$.

We will implicitly use the following facts concerning quasisimilarity throughout this paper.

LemMA. Suppose $A: H \rightarrow H, B: H^{\prime} \rightarrow H^{\prime}$ and $X: H \rightarrow H^{\prime}$ are operators such that $X$ has dense range and $X A=B X$. If $A$ is $n$-cyclic, then $B$ is cyclic of order at most $n$.

Corollary. If $A \sim \sim B$ and $A$ is $n$-cyclic, then $B$ is also $n$-cyclic.

Let $\mathcal{E}$ be a subset of $H$. The smallest invariant subspace of $A: H \rightarrow H$ which contains $\mathcal{E}$ is denoted $\Re(\mathcal{E} ; A)$. Similarly, the smallest subspace of $H$ which contains $\mathcal{E}$ and reduces $A$ is denoted $\Re(\mathcal{E} ; A)$. If $\mathcal{E}=\left\{x_{1}, \ldots, x_{n}\right\}$ is finite, then we write $\mathscr{N}\left(x_{1}, \ldots, x_{n} ; A\right)$ and $\Re\left(x_{1}, \ldots, x_{n} ; A\right)$ for $\Re(\mathcal{E}, A)$ and $\Re(\mathcal{E}, A)$, respectively. 
By measure we will always mean a positive, finite measure on the Borel subsets of $\mathbf{C}$. We will say that a measure $\mu$ is carried by a Borel set $E$ if $\mu(C \backslash E)=0$. The support of a measure $\mu$, denoted supp $\mu$, is the complement of the union of all open sets of $\mu$-measure zero. The measure $\mu$ restricted to a Borel set $E$ is the measure $\left.\mu\right|_{E}$ defined by $\left.\mu\right|_{E}(F)=\mu(E \cap F)$. We will be particularly interested in measures which are carried by $\bar{D}$, where $D=\{z$ : $|z|<1\}$. If $\mu$ is such a measure, then we will write $\mu_{0}$ for $\left.\mu\right|_{\text {F. }}$. (Recall that $\mathscr{T}$ is the boundary of $D$.)

Let $\mu$ be a measure with compact support. Let $W_{\mu}: L^{2}(\mu) \rightarrow L^{2}(\mu)$ be the operation of "multiplication by $z$ ". Thus $\left(W_{\mu} f\right)(z)=z f(z)$ for each $f \in$ $L^{2}(\mu)$. Up to unitary equivalence the operator $W_{\mu}$ is the most general cyclic normal operator. For $f$ and $g$ in $L^{2}(\mu)$ we will write $(f, g)_{\mu}$ for their inner product and $\|f\|_{\mu}$ for the $L^{2}(\mu)$-norm of $f$.

2. Normal operators. We collect here some properties of normal operators which we will use in later sections. The above representation for cyclic normal operators generalizes to the noncyclic case as follows.

Let $\mu=\left(\mu_{1}, \ldots, \mu_{n}\right)$ be a finite sequence of measures. Let $F=$ $\left(f_{1}, \ldots, f_{n}\right)$ and $G=\left(g_{1}, \ldots, g_{n}\right)$ be measurable functions mapping $\mathbf{C}$ into $\mathrm{C}^{n}$. We will say that $F=G$ a.e. $[\mu]$ if $f_{j}=g_{j}$ a.e. $\left[\mu_{j}\right], 1<j<n$. This defines an equivalence relation on the set of such functions, and, following the usual custom, we will ignore the distinction between a function and the equivalence class to which it belongs. Let $L^{2}(\mu)$ be the set of (equivalence classes of) measurable functions $F: \mathrm{C} \rightarrow \mathrm{C}^{n}$ such that

$$
\left\{\sum_{k=1}^{n} \int\left|f_{k}\right|^{2} d \mu_{k}\right\}^{1 / 2}<\infty
$$

where $F=\left(f_{1}, \ldots, f_{n}\right)$. Using the left-hand side of (1) as norm, $L^{2}(\mu)$ becomes a separable Hilbert space. Define an operator $W_{\mu}: L^{2}(\mu) \rightarrow L^{2}(\mu)$ by $\left(W_{\mu} F\right)(z)=z F(z)=\left(z f_{1}(z), \ldots, z f_{n}(z)\right)$.

These definitions may also be made for an infinite sequence of measures $\mu=\left(\mu_{1}, \mu_{2}, \ldots\right)$. In this case we consider functions $F=\left(f_{1}, f_{2}, \ldots\right)$ mapping $C$ into the space of sequences of complex numbers and assume each $f_{j}$ is measurable.

LEMMA 2.1. A normal operator $T: K \rightarrow K$ is $n$-cyclic if, and only if, $n$ is the smallest integer for which there exist $n$ vectors $x_{1}, \ldots, x_{n} \in K$ such that $K=\Re\left(x_{1}, \ldots, x_{n} ; T\right)$.

Proof. Suppose $T$ is $n$-cyclic and suppose $K=\Re\left(x_{1}, \ldots, x_{j} ; T\right)$. Let $\tilde{x}_{1}=x_{1}$, and let $\tilde{x}_{i}$ be the orthogonal projection of $x_{i}$ into

$$
K \ominus \Re\left(x_{1}, \ldots, x_{i-1} ; T\right), \quad 2<i<j .
$$


Choose $y_{i}$ such that $\Re\left(y_{i} ; T\right)=\Re\left(\tilde{x}_{i} ; T\right)$ (cf. [1, Theorem 6]). We have

$$
\Re\left(y_{1}, \ldots, y_{j} ; T\right) \supset \Re\left(\tilde{x}_{1} ; T\right) \oplus \cdots \oplus \Re\left(\tilde{x}_{j} ; T\right)=K \text {. }
$$

Therefore, $n<j$.

LEMMA 2.2. Let $\mu=\left(\mu_{1}, \ldots, \mu_{n}\right)$ be a sequence of measures. The following statements are equivalent:

(1) $W_{\mu}$ is $n$-cyclic;

(2) there exists a nonzero measure $\nu$ such that $\nu \ll \mu_{j}, 1<j<n$;

(3) there exists a Borel set $E$ such that $\mu_{j}(E)>0$ and $\left.\mu_{j}\right|_{E} \ll \mu_{k}, 1<j$, $k<n$.

Proof. Suppose $W_{\mu}$ is $n$-cyclic. For each $j, 1<j<n-1$, we may write $\mu_{n}=\nu_{j a}+\nu_{j s}$, where $\nu_{j a} \ll \mu_{j}$ and $\nu_{j s} \perp \mu_{j}$. Let $E_{j}$ be a Borel set such that $\nu_{j s}\left(C \backslash E_{j}\right)=\mu_{j}\left(E_{j}\right)=0$, and set $E_{j}^{\prime}=\operatorname{supp} \mu_{j} \backslash E_{j}$. Let

$$
F_{j}=\left(0, \ldots, 0, \chi_{E_{j}}, 0, \ldots, 0, \chi_{E_{j}}\right), \quad 1<j<n-1 .
$$

Both $(0, \ldots, 0,1,0, \ldots, 0)$ and $\left(0, \ldots, 0, \chi_{E_{j}}\right)$ are in $\Re\left(F_{j} ; W_{M}\right)$. Let

$$
E=\bigcup_{j=1}^{n-1} E_{j}
$$

It follows that $(1,0, \ldots, 0),(0,1,0, \ldots, 0), \ldots,(0, \ldots, 0,1,0)$ and $\left(0, \ldots, 0, \chi_{E}\right)$ are all elements of $\Re\left(F_{1}, \ldots, F_{n-1} ; W_{\mu}\right)$. Since $\Re\left(F_{1}, \ldots, F_{n-1} ; W_{\mu}\right) \neq L^{2}(\mu)$, we must conclude that $\mu_{n}(C \backslash E)>0$. By construction, $\nu_{j s}(C \backslash E)=0,1<j<n-1$. Therefore, the measure $\left.\mu_{n}\right|_{C \backslash E}$ satisfies statement (2).

Now suppose the measure $\nu$ satisfies (2). Let $E$ be a Borel set such that $\nu$ is carried by $E$ and $\nu$ and $\left.\mu_{j}\right|_{E}$ are mutually absolutely continuous. Then

$$
\left.\left.\mu_{j}\right|_{E} \ll \nu \ll \mu_{k}\right|_{E} \ll \mu_{k}
$$

and $\mu_{j}(E)>0$ because $\nu(E)>0$.

Finally, suppose there is a Borel set E satisfying statement (3). Let $\nu=\left.\mu_{1}\right|_{E}$ and note that $\left.\mu_{j}\right|_{E}$ and $\nu$ are mutually absolutely continuous. Suppose $L^{2}(\mu)$ $=\Re\left(F_{1}, \ldots, F_{n^{\prime}} ; W_{\mu}\right)$. As shown in the proof of Lemma 2.1, we may assume that

$$
\Re\left(F_{j} ; W_{\mu}\right) \perp \Re\left(F_{k} ; W_{\mu}\right), \quad j \neq k .
$$

Because of this orthogonality condition we may conclude that there are measurable functions $g_{j k}$ such that

$$
g_{j 1} F_{1}+\cdots+g_{j, n^{\prime}} F_{n^{\prime}}=(0, \ldots, 0,1,0, \ldots, 0), \quad 1<j<n
$$

(the " 1 " appearing in the $j$ th position). Let $A(z)$ be the matrix $\left(g_{i j}(z)\right)$. Then the equations (2) imply that $A(z)$, considered as a linear transformation from $\mathbf{C}^{n^{\prime}}$ into $\mathbf{C}^{n}$, is surjective for almost every (with respect to $\nu$ ) $z$. This is possible only if $n^{\prime}>n$. Therefore $W_{\mu}$ is $n$-cyclic by Lemma 2.1 . 
THEOREM 2.3. Let $T: K \rightarrow K$ be a normal operator on a separable Hilbert space $K$. Then there exists a sequence of measures $\mu=\left(\mu_{1}, \mu_{2}, \ldots\right)$ such that $\mu_{1} \gg \mu_{2} \gg \ldots$ and $T \cong W_{\mu}$. If $T$ is cyclic of finite order $n$, then we may take $\boldsymbol{\mu}=\left(\mu_{1}, \ldots, \mu_{n}\right)$.

Proof. See [2, Theorem 10.3]. The last statement of the theorem follows from Lemma 2.2.

Let $T: K \rightarrow K$ be normal. One result of the spectral theorem is that for each $x \in K$ there exists a unique measure $\nu_{x}$ such that

$$
\left(T^{j} x, T^{k} x\right)=\int z^{j} \bar{z}^{k} d \nu_{x}(z) .
$$

In addition, there is a unitary operator $V: K \rightarrow L^{2}\left(\nu_{x}\right)$ such that $V x=1$ and

$$
\left.T\right|_{x(x ; T)} \approx W_{y_{x}} \text { via } V \text {. }
$$

If $K$ is separable, the measure $\nu_{x}$ may be found as follows.

LEMMA 2.4. Let $T: K \rightarrow K$ be a normal operator on a separable Hilbert space. Suppose $T \simeq W_{\mu}$ via the unitary operator $V$, where $\mu=\left(\mu_{1}, \mu_{2}, \ldots\right)$. If $V x=\left(f_{1}, f_{2}, \ldots\right)$, then $\nu_{x}$ is defined by

$$
d \nu_{x}=\sum_{j=1}^{\infty}\left|f_{j}\right|^{2} d \mu_{j}
$$

In particular, if $\mu_{1} \gg \mu_{2} \gg \ldots$, then $\nu_{x} \ll \mu_{1}$.

3. Subnormal operators. We now turn to the relationship between a subnormal operator and its minimal normal extension. Suppose $S: H \rightarrow H$ is subnormal and $n$-cyclic with minimal normal extension $T: K \rightarrow K$. It is easy to show (see Theorem 3.2) that $T$ is $n^{\prime}$-cyclic, where $n^{\prime}<n$. That $n^{\prime}$ can be smaller than $n$ is demonstrated dramatically in the following

EXAMPLE 3.1. Let $\tau$ be Lebesgue area measure restricted to $D$, and let $H_{n}=\mathfrak{R}\left(1,|z|^{2}, \ldots,|z|^{2 n} ; W_{\tau}\right)$. Note that if $s \neq j$, then $\left(z^{j}|z|^{2 k}, z^{s}|z|^{2 t}\right)_{\tau}=0$ for $0<k, t<n$. Let $T_{n}=\left.W_{\tau}\right|_{H_{n}}$. It is easily checked that $\left\{|z|^{2 k}\right\}_{k=0}^{n}$ spans ker $T_{n}^{*}$. Hence, $\left(\operatorname{ran} T_{n}\right)^{-}$has codimension $n+1$, and it follows that $T_{n}$ is $(n+1)$-cyclic. On the other hand, the minimal normal extension is cyclic. If

$$
H_{\infty}=\left(\bigcup_{n=0}^{\infty} H_{n}\right)^{-} \text {and } T_{\infty}=W_{\tau} \mid H_{\infty},
$$

then $T_{\infty}$ is a subnormal operator which is not finitely cyclic $\left(|z|^{2 k} \in \operatorname{ker} T_{\infty}^{*}\right.$ for each $k>0$ ) even though its minimal normal extension is cyclic.

In view of Theorem 2.3 , the subnormal operator $S$ is unitarily equivalent to the restriction of $W_{\mu}$ to a subspace $\tilde{H}$ of $L^{2}(\mu)$ for some sequence of measures $\mu=\left(\mu_{1}, \ldots, \mu_{n^{\prime}}\right)$. Since $S$ is $n$-cyclic, there exist $n$ elements $F_{1}, \ldots, F_{n}$ of $\tilde{H}$ such that $\tilde{H}=\mathscr{T}\left(F_{1}, \ldots, F_{n} ; W_{\mu}\right)$. In order that this information be useful, we must know something more about $F_{1}, \ldots, F_{n}$. 
THEOREM 3.2. Suppose $S: H \rightarrow H$ is subnormal and $n$-cyclic with minimal normal extension $T: K \rightarrow K$. Then $T$ is $n^{\prime}$-cyclic, where $n^{\prime}<n$. Furthermore, there exist a sequence of measures $\mu=\left(\mu_{1}, \ldots, \mu_{n}\right)$ and elements $F_{1}, \ldots, F_{n}$ of $L^{2}(\mu)$ with $\mu_{k+1} \ll \mu_{k}, 1<k<n^{\prime}$, and

$$
\begin{aligned}
F_{1}= & (1,0, \ldots, 0), \\
F_{2}= & \left(\psi_{21}, 1,0, \ldots, 0\right), \\
& \vdots \\
F_{n^{\prime}}= & \left(\psi_{n^{\prime} 1}, \ldots, \psi_{n^{\prime}, n^{\prime}-1}, 1\right),
\end{aligned}
$$

such that $\left.S \simeq W_{\mu}\right|_{\tilde{H}}$, where $\tilde{H}=\operatorname{TR}\left(F_{1}, \ldots, F_{n} ; W_{\mu}\right)$.

Proof. Choose $n$ vectors $x_{1}, \ldots, x_{n}$ in $H$ such that $H=\mathscr{T}\left(x_{1}, \ldots, x_{n}\right.$; $S$ ). Since $T$ is the minimal normal extension of $S$, we have $K=$ $\Re\left(x_{1}, \ldots, x_{n} ; T\right)$. By Lemma 2.1,T is $n^{\prime}$-cyclic, where $n^{\prime}<n$. Define $\tilde{x}_{k}$ as in the proof of Lemma 2.1. Of course, one of the vectors $\tilde{x}_{k}$ may be zero. On the other hand, by reordering the set $\left\{x_{1}, \ldots, x_{n}\right\}$ if necessary, we may assume that $\tilde{x}_{1}, \ldots, \tilde{x}_{n^{\prime \prime}}$ are not zero, while (if $n^{\prime \prime}<n$ ) $\tilde{x}_{n^{\prime \prime}+1}=\cdots=\tilde{x}_{n}=$ 0 . For $1<k<n^{\prime \prime}$, there exist a measure $\mu_{k}$ and a unitary operator $V_{k}: \Re\left(\tilde{x}_{k}\right.$; $T) \rightarrow L^{2}\left(\mu_{k}\right)$ such that $V_{k} \tilde{x}_{k}=1$ and $\left.T\right|_{\text {ou }\left(\tilde{x}_{k} ; T\right)} \approx W_{\mu_{k}}$ via $V_{k}$. Let $\mu=$ $\left(\mu_{1}, \ldots, \mu_{n^{*}}\right)$ and define $V: K \rightarrow L^{2}(\mu)$ by $V\left(y_{1} \oplus \cdots \oplus y_{n^{\prime \prime}}\right)=$ $\left(V_{1} y_{1}, \ldots, V_{n^{n}} y_{n^{*}}\right)$, so that $T \cong W_{\mu}$ via $V$. Let $F_{k}=V x_{k}$. By construction $F_{1}, \ldots, F_{n^{\prime \prime}}$ have the form of (3) with $n^{\prime}$ replaced by $n^{\prime \prime}$. If $\tilde{H}=$ $\operatorname{TC}\left(F_{1}, \ldots, F_{n} ; W_{\mu}\right)$, then $\left.S \simeq W_{\mu}\right|_{\tilde{H}}$.

For arbitrary choice of the set $\left\{x_{1}, \ldots, x_{n}\right\}$ it may happen that $n^{\prime \prime}>n^{\prime}$ (see the following example). We will show, however, that the set $\left\{x_{1}, \ldots, x_{n}\right\}$ could have been chosen in such a way that $\mu_{k+1} \ll \mu_{k}, 1<k<n^{\prime \prime}$. This assumption will imply (Lemma 2.2) that $W_{\mu}$ is $n^{\prime \prime}$-cyclic. Since $T$ is $n^{\prime}$-cyclic, we will have $n^{\prime}=n^{\prime \prime}$, and the proof will be complete.

The basic idea is first to modify $x_{1}$ so that with its new value, $\mu_{k} \ll \mu_{1}$, $2<k<n^{\prime \prime}$. Next, $x_{2}$ is modified so that $\mu_{k} \ll \mu_{2}, 3<k<n^{\prime \prime}$, and so on. We will illustrate this procedure for this case $n^{\prime \prime}=3$. Choose $\alpha_{3} \neq 0$ such that $\left|1+\alpha_{3} \psi_{32}\right|>0$ a.e. $\left[\mu_{2}\right]$, and then choose $\alpha_{2} \neq 0$ such that $\mid 1+\alpha_{2}\left(\psi_{21}+\right.$ $\left.\alpha_{3} \psi_{31}\right) \mid>0$ a.e. $\left[\mu_{1}\right]$. Now, replace $x_{1}$ by $x_{1}+\alpha_{2} x_{2}+\alpha_{2} \alpha_{3} x_{3}$. This has the effect of replacing $\mu_{1}$ by $\mu_{1}^{\prime}$ where

$$
d \mu_{1}^{\prime}=\left|1+\alpha_{2} \psi_{21}+\alpha_{2} \alpha_{3} \psi_{31}\right|^{2} d \mu_{1}+\left|\alpha_{2}\right|^{2}\left|1+\alpha_{3} \psi_{32}\right|^{2} d \mu_{2}+\left|\alpha_{2} \alpha_{3}\right|^{2} d \mu_{3} .
$$

This replacement will also effect the definitions of $\tilde{x}_{2}$ and $\tilde{x}_{3}$ as well as the measures $\mu_{2}$ and $\mu_{3}$ and the functions $\psi_{j k}$. The important fact is that with the new definitions of $\mu_{1}, \mu_{2}$ and $\mu_{3}$ we have $\mu_{2} \ll \mu_{1}$ and $\mu_{3} \ll \mu_{1}$.

If $n^{\prime}>1$, we may still assume that $\tilde{x}_{2} \neq 0$ by interchanging $x_{2}$ and $x_{3}$ if necessary. Choose $\beta_{3} \neq 0$ such that $\left|1+\beta_{3} \psi_{32}\right|>0$ a.e. $\left[\mu_{2}\right]$, and replace $x_{2}$ 
by $x_{2}+\beta_{3} x_{3}$. This has the effect of replacing $\tilde{x}_{2}$ by $V^{*}\left(0,1+\beta_{3} \psi_{32}, \beta_{3}\right)$, and hence of replacing $\mu_{2}$ by $\mu_{2}^{\prime}$, where

$$
d \mu_{2}^{\prime}=\left|1+\beta_{3} \psi_{32}\right|^{2} d \mu_{2}+\left|\beta_{3}\right|^{2} d \mu_{3} \text {. }
$$

Thus we have $\mu_{1} \gg \mu_{2} \gg \mu_{3}$.

The following example shows that we can have $n^{\prime}<n^{\prime \prime}$ in the above proof.

EXAMPLE. Let $E_{1}=\left\{z:|z|<\frac{2}{3}\right\}$, let $E_{2}=\left\{z: \frac{1}{3}<|z|<1\right\}$, and set $g_{i}=$ $\chi_{E_{i}}, i=1,2$. Let $H=\mathfrak{T}\left(g_{1}, g_{2} ; W_{\tau}\right)(\tau$ is defined in Example 3.1), and set $S=\left.W_{\tau}\right|_{H}$. Then $g_{i} \in \operatorname{ker} S^{*}, i=1,2$; hence, $S$ is 2-cyclic with cyclic normal extension $W_{\tau}$. On the other hand, if we let $x_{i}=g_{i}$ in the above proof, then we have $n^{\prime \prime}=2$.

The pair $\left(\mu ; F_{1}, \ldots, F_{n}\right)$ will be called a standard representation for the $n$-cyclic subnormal operator $S$ if

(1) $\mu=\left(\mu_{1}, \ldots, \mu_{n^{\prime}}\right)$ is a sequence of measures with $n^{\prime}<n$ and $\mu_{1} \gg \mu_{2}$ $\gg \cdots \gg \mu_{n^{\prime}}$

(2) $F_{1}, \ldots, F_{n}$ are elements of $L^{2}(\mu)$ with $F_{1}, \ldots, F_{n^{\prime}}$ having the form (3); and

(3) $S$ is unitarily equivalent to $\left.W_{\mu}\right|_{\tilde{H}}$, where $\tilde{H}=\mathfrak{T}\left(F_{1}, \ldots, F_{n} ; W_{\mu}\right)$.

In view of the previous theorem, every $n$-cyclic subnormal operator has a standard representation. One obvious example of an $n$-cyclic subnormal operator is $S=U_{\mu_{1}} \oplus U_{\mu_{2}} \oplus \cdots \oplus U_{\mu_{n}}$, where $\mu_{j}$ is a measure with compact support, $1<j<n$, and $\mu_{1} \gg \mu_{2} \gg \cdots \gg \mu_{n}$. Let $\mu=\left(\mu_{1}, \ldots, \mu_{n}\right)$ and let $F_{j}=(0, \ldots, 0,1,0, \ldots, 0)$ (the " 1 " appearing in the $j$ th position). A standard representation for $S$ is $\left(\mu ; F_{1}, \ldots, F_{n}\right)$. Let $H^{2}(\mu)=\operatorname{TR}\left(F_{1}, \ldots, F_{n}\right.$; $W_{\mu}$ ) and let $U_{\mu}=\left.W_{\mu}\right|_{H^{2}(\mu)}$ so that $S \cong U_{\mu}$. In particular, if $\mu_{j}=m, 1<j<$ $n$, then we will write $\mathbf{m}^{n}$ for $\mu$. The operator $U_{\mathbf{m}^{n}}$ is just the unilateral shift of multiplicity $n$. We will study the subnormal operators quasisimilar to $U_{\mathbf{m}^{n}}$ in the next section.

CoRollary 3.3. Suppose $S: H \rightarrow H$ is subnormal and finitely cyclic with minimal normal extension $T: K \rightarrow K$. Then there exists $x \in H$ such that $\nu_{y} \ll \nu_{x}$ for each $y \in K$.

Proof. Let $\left(\mu ; F_{1}, \ldots, F_{n}\right)$ be a standard representation for $S$, where $\mu=\left(\mu_{1}, \ldots, \mu_{n^{\prime}}\right)$. By Lemma 2.4 , we have $\nu_{G} \ll \mu_{1}$ for each $G \in L^{2}(\mu)$. But $\nu_{F_{1}}=\mu_{1}$.

For a beautiful proof of the following proposition, see Morrel [11].

Proposimon 3.4. If $T: H \rightarrow H$, then $T$ has reducing subspaces $H_{0}$ and $H_{1}$ such that $\left.T\right|_{H_{0}}$ is normal, $\left.T\right|_{H_{1}}$ is completely nonnormal, and $H=H_{0} \oplus H_{1}$. Furthermore,

$$
H_{0}=\bigcap_{j, k>0} \operatorname{ker}\left(T^{j} T^{* k}-T^{* k} T^{j}\right)
$$


Proposition 3.4 yields a canonical decomposition of an operator $T$ into a normal part and a completely nonnormal part. We will call this decomposition the normal decomposition of $T$. We should mention that a completely nonnormal subnormal operator is usually said to be completely subnormal or pure.

An operator $S$ is said to be dominant (cf. [12]) if $\operatorname{ran}(S-\lambda) \subset \operatorname{ran}(S-\lambda)^{*}$ for each $\lambda \in \mathbf{C}$. In particular, every hyponormal operator is dominant.

Proposirion 3.5. Let $S$ and $T$ be dominant operators with normal decompositions $S_{0} \oplus S_{1}$ on $H=H_{0} \oplus H_{1}$ and $T_{0} \oplus T_{1}$ on $K=K_{0} \oplus K_{1}$, respectively. Suppose there exist operators $X: H \rightarrow K$ and $Y: K \rightarrow H$ such that $X$ and $Y$ have no kernel, $X S=T X$, and $S Y=Y T$. Then $S_{0} \simeq T_{0}$.

Proof. Let

$$
\begin{array}{ll}
H_{2}=\left(Y K_{0}\right)^{-}, & K_{2}=\left(X H_{0}\right)^{-}, \\
X_{0}=\left.X\right|_{H_{0}}: H_{0} \rightarrow K_{2}, & Y_{0}=\left.Y\right|_{K_{0}}: K_{0} \rightarrow H_{2}, \\
S_{2}=\left.S\right|_{H_{2}}, & T_{2}=\left.T\right|_{K_{2}} .
\end{array}
$$

By [12, Theorem 1] $S_{2}$ and $T_{2}$ are normal. From [12, Lemma 2] it follows that $H_{2}$ and $K_{2}$ reduce $S_{2}$ and $T_{2}$, respectively. Therefore, $H_{2} \subset H_{0}$ and $K_{2} \subset K_{0}$. Since $X_{0}$ and $Y_{0}$ have no kernel, from [5, Lemma 4.1] it follows that $S_{0} \simeq T_{2}$ and $T_{0} \simeq S_{2}$. Hence [9] $S_{0} \simeq T_{0}$.

Let $T: K \rightarrow K$ be a normal operator on a separable Hilbert space with spectral measure $\mathbf{P}$ so that $T=\int z d \mathbf{P}(z)$. Let $\mu=\left(\mu_{1}, \mu_{2}, \ldots\right)$ be a sequence of measures, and let $V: K \rightarrow L^{2}(\mu)$ be a unitary operator such that $T \simeq W_{k}$ via $V$. Let $L_{E}^{2}(\mu)$ be those elements $F$ of $L^{2}(\mu)$ for which $F=0$ a.e. [ $\mu$ ] on $\mathrm{C} \backslash E$. Then $\mathrm{P}(E)$ (or, more precisely, $V \mathrm{P}(E) V^{*}$ ) is the projection of $L^{2}(\mu)$ onto $L_{E}^{2}(\mu)$. In particular, $E$ is a set of spectral measure zero for $T$ if, and only if, $\mu_{j}(E)=0$ for each $j>1$.

A contraction $A$ operating on $H$ is said to be completely nonunitary (c.n.u.) if no subspace of $H$ reduces $A$ to a unitary operator.

Proposition 3.6. Suppose $S$ is a c.n.u. subnormal contraction on a separable Hilbert space $H$ with minimal normal extension $T: K \rightarrow K$. If $E \subset \mathcal{T}$ is a set of Lebesgue measure zero, then $E$ is a set of spectral measure zero for $T$.

Proof. First, suppose $S$ is $n$-cyclic with standard representation ( $\mu$; $\left.F_{1}, \ldots, F_{n}\right)$, where $\mu=\left(\mu_{1}, \ldots, \mu_{n}\right)$. Since

$$
\left.W_{\mu}\right|_{\Re\left(F_{1} ; W_{\mu}\right)} \simeq U_{\mu_{1}}
$$

the operator $U_{\mu_{1}}$ is c.n.u. By a result of Clary [4, Lemma 4.5] this is possible only if $\mu_{1} \ll m$. Therefore, $\left.\mu_{j}\right|_{g} \ll m$ because $\mu_{j} \ll \mu_{1}$. The proof is complete for the finitely cyclic case. 
For the general case, let $\left\{e_{1}, e_{2}, \ldots\right\}$ be an orthonormal basis of $H$, let $f_{1}=e_{1}$, and let $f_{k}$ be the orthogonal projection of $e_{k}$ into $K \Theta$ $\Re\left(e_{1}, \ldots, e_{k-1} ; T\right)$, so that $K=\Re\left(f_{1} ; T\right) \oplus \Re\left(f_{2} ; T\right) \oplus \ldots$ We must show that $\left.\nu_{f_{k}}\right|_{S} \ll m$. Let $H_{n}=\mathscr{T}\left(e_{1}, \ldots, e_{n} ; S\right)$, and let $S_{n}=\left.S\right|_{H_{n}}$. The minimal normal extension of $S_{n}$ is $\left.T\right|_{K_{n}}$, where

$$
K_{n}=\Re\left(e_{1}, \ldots, e_{n} ; T\right)=\Re\left(f_{1} ; T\right) \oplus \cdots \oplus \Re\left(f_{n} ; T\right) .
$$

By the first part of this proof, $\left.\nu_{f_{k}}\right|_{g} \ll m, 1<k<n$. Since $n$ was arbitrary, the proof is complete.

4. Subnormal operators of type $\delta_{n}$. We now come to the main results. Suppose $S$ and $S^{\prime}$ are quasisimilar subnormal operators. In the previous section we showed that their normal parts must be unitarily equivalent. In this section we find additional hypotheses which imply that their completely subnormal parts must be quasisimilar. A complete characterization of those subnormal operators quasisimilar to a unilateral shift of finite multiplicity is also given in Theorem 4.4 .

Proposition 4.1. Let $S: H \rightarrow H$ be a subnormal operator with standard representation $\left(\mu ; F_{1}, \ldots, F_{n}\right)$, where $\mu=\left(\mu_{1}, \ldots, \mu_{n^{\prime}}\right)$. Suppose supp $\mu_{j} \subseteq \bar{D}$ and $\left.\mu_{j}\right|_{g} \ll m, 1<j<n^{\prime}$. In addition, suppose there is a bounded operator $X$ : $H \rightarrow H^{2}\left(\mathrm{~m}^{n}\right)$ with dense range satisfying $X S=U_{\mathrm{m}^{\mathrm{m}}} X$. Then

(a) $n^{\prime}=n$,

(b) $X$ is one-to-one,

(c) $\mu_{\left.j\right|_{5}}$ and $m$ are mutually absolutely continuous.

Proof. Let $\mu_{0}=\left(\mu_{10}, \ldots, \mu_{n^{\prime} 0}\right)$, where $\mu_{j 0}=\left.\mu_{j}\right|_{g}$. Let $H_{0}=$ $\operatorname{TR}\left(F_{1}, \ldots, F_{n} ; W_{\mu_{0}}\right)$, and set $S_{0}=\left.W_{\mu_{0}}\right|_{H_{0}}$. As in $[4$, Lemma $4.5 \gamma]$, the operator $X$ induces an operator $X_{0}: H_{0} \rightarrow H^{2}\left(\mathrm{~m}^{n}\right)$ with $X_{0} F=X F$ for each $F \in \mathscr{T}\left(F_{1}, \ldots, F_{n} ; W_{\mu}\right)$. Now $X_{0}$ has an extension $\tilde{X}_{0}: L^{2}\left(\mu_{0}\right) \rightarrow L^{2}\left(\mathrm{~m}^{n}\right)$ such that $\left.\tilde{X}_{0}\right|_{H_{0}}=X_{0}$ and $\tilde{X}_{0} W_{\mu_{0}}=W_{\mathbf{w}^{n}} \tilde{X}_{0}$ (cf. [5, Corollary 5.11). By a lemma of Douglas [5, Lemma 4.1], $\left(\operatorname{ker} \tilde{X}_{0}\right)^{\perp}$ reduces $W_{\mu_{0}},\left(\operatorname{ran} \tilde{X}_{0}\right)^{-}$reduces $W_{\mathbf{m}^{n}}$, and

$$
\left.\left.W_{\mu_{0}}\right|_{\left(\operatorname{ker} \tilde{X}_{0}\right)^{\perp}} \simeq W_{\operatorname{mon}}\right|_{\left(\operatorname{ran} \tilde{x}_{0}\right)^{-}} .
$$

But $\left(\operatorname{ran} \tilde{X}_{0}\right)^{-} \supset H^{2}\left(\mathbf{m}^{n}\right)$, and so $\left(\operatorname{ran} \tilde{X}_{0}\right)^{-}=L^{2}\left(\mathbf{m}^{n}\right)$.

Suppose $F \in \operatorname{ker} \tilde{X}_{0}, F \neq 0$. Let $K=\Re\left(F ; W_{\mu_{0}}\right)$. Then

$$
\left.\left.\left.W_{\mu_{0}}\right|_{\left(\operatorname{ker} \tilde{x}_{0}\right)} ^{\perp} \oplus W_{\mu_{0}}\right|_{K} \simeq W_{\mathbf{m}^{n}} \oplus W_{\mu_{0}}\right|_{K} .
$$

But $\nu_{F} \ll m$; therefore $\left.W_{\text {man }^{n}} \oplus W_{p_{0}}\right|_{K}$ is $(n+1)$-cyclic by Lemma 2.2. This contradiction shows that $\tilde{X}_{0}$ and, a fortiori, $X$ have no kernel. Thus, $W_{n_{0}} \simeq$ $W_{\mathrm{m}^{n}}$ and $n^{\prime}=n$. It now follows that $\mu_{\left.j\right|_{s}}$ and $m$ are mutually absolutely continuous. 
Suppose an operator $S$ is subnormal and $n$-cyclic with standard representation $\left(\mu ; F_{1}, \ldots, F_{n}\right)$, where $\mu=\left(\mu_{1}, \ldots, \mu_{n^{\prime}}\right)$. The operator $S$ is said to be of type $\delta_{n}$ if and only if $n^{\prime}=n$ and $\mu_{j}$ is of type $\delta, 1<j<n$. The next two lemmas together show that if $S$ is of type $\delta_{n}$, then $S$ is quasisimilar to the unilateral shift of multiplicity $n$.

Lemma 4.2. Suppose $S: H \rightarrow H$ is a subnormal operator of type $\mathcal{S}_{n}$. Then there is a quasiinvertible operator $X: H \rightarrow H^{2}\left(m^{n}\right)$ such that $X S=U_{m} X$.

Proof. Let $\left(\mu ; F_{1}, \ldots, F_{n}\right)$ be a standard representation for $S$, where

$$
F_{k}=\left(\psi_{k 1}, \ldots, \psi_{k, k-1}, 1,0, \ldots, 0\right) \text {. }
$$

Let $\tilde{H}=\mathscr{T}\left(F_{1}, \ldots, F_{n} ; W_{\mu}\right)$ and set $\tilde{S}=\left.W_{\mu}\right|_{\tilde{H}}$. For convenience, let $\psi_{k k}=$ 1 and $\psi_{k j}=0$ for $j>k, 1<k<n$. We assert that there exist outer functions $g_{1}, \ldots, g_{n}$ in $H^{2}$ such that for any set of $n$ polynomials $\left\{p_{1}, \ldots, p_{n}\right\}$ we have

$$
\left|p_{i} g_{i}\right|^{2}<\sum_{j=1}^{n}\left|\sum_{k=j}^{n} p_{k} \psi_{k j}\right|^{2} \frac{d \mu_{j}}{d m} \quad \text { a.e. }[m], \quad 1<i<n \text {. }
$$

Suppose for now that this assertion is true. Define $G_{1}, \ldots, G_{n} \in H^{2}\left(\mathrm{~m}^{n}\right)$ by $G_{k}=\left(0, \ldots, 0, g_{k}, 0, \ldots, 0\right)$, the $g_{k}$ appearing in the $k$ th position. Then we may define $X: \tilde{H} \rightarrow H^{2}\left(\mathrm{~m}^{n}\right)$ by $X F_{k}=G_{k}$ and $X \tilde{S}=U_{\mathrm{m}^{n}} X$. A straightforward computation shows that this defines a bounded operator. Since $g_{1}, \ldots, g_{n}$ are outer, $X$ has dense range. Suppose $\left(f_{1}, \ldots, f_{n}\right) \in \tilde{H}$ and $X\left(f_{1}, \ldots, f_{n}\right)=0$. Choose polynomials $p_{j k}, 1<k<n, j>1$, such that $\sum_{k=1}^{n} p_{j k} F_{k} \rightarrow\left(f_{1}, \ldots, f_{n}\right)$ in $\tilde{H}$ and such that $X\left(p_{j 1} F_{1}+\cdots+p_{j n} F_{n}\right) \rightarrow 0$ pointwise a.e. $\left[\mathrm{m}^{n}\right]$. This means that $p_{j k} g_{k} \rightarrow 0$ pointwise a.e. $[\mathrm{m}]$ on $\mathcal{J}$ and $p_{j k} g_{k} \rightarrow 0$ pointwise everywhere in $D$. Since $g_{k}$ is outer, this is possible only if $\sum_{k=1}^{n} p_{j k} F_{k} \rightarrow 0$ pointwise a.e. $[\mu]$; that is, $\left(f_{1}, \ldots, f_{n}\right)=0$.

We now prove the assertion above by induction on $n$. If $n=1$, let $g_{1}$ be an outer function with $\left|g_{1}\right|^{2}=d \mu_{1} / d m$. Suppose $n>2$ and suppose the result is true with $n-1$ in place of $n$. Then there are outer functions $\tilde{g}_{1}, \ldots, \tilde{g}_{n-1}$ in $H^{2}$ such that

$$
\left|p_{i} \tilde{g}_{i}\right|^{2}<\sum_{j=1}^{n-1}\left|\sum_{k=j}^{n-1} p_{k} \psi_{k j}\right|^{2} \frac{d \mu_{j}}{d m} \quad \text { a.e. }[m]
$$

for any set of $(n-1)$ polynomials $p_{1}, \ldots, p_{n-1}$. Let $g_{n}$ be an outer function such that $\left|g_{n}\right|^{2}=d \mu_{n} / d m$. For $1<i<n-1$, let

$$
\varphi_{i}=\left|\tilde{g}_{i}\right|^{2} \min \left\{\frac{1}{2 n-1},\left[2(n-1) \sum_{j=1}^{n-1}\left|\psi_{n j}\right|^{2} \frac{d \mu_{j}}{d m}\right]^{-1} \frac{d \mu_{n}}{d m}\right\}
$$

Then $\int \log \varphi_{\mathrm{i}} d m>-\infty$. Indeed, fix $i, 1<i<n-1$, and let

$$
E=\left\{z \in \mathcal{T}: \varphi_{i}(z)=\frac{1}{2 n-1}\left|\tilde{g}_{i}(z)\right|^{2}\right\}
$$


Then

$$
\int_{E} \log \varphi_{i} d m=\int_{E} \log \frac{\left|\tilde{g}_{i}\right|^{2}}{2 n-1} d m>-\infty
$$

while

$$
\begin{aligned}
\int_{\mathscr{T} \backslash E} \log \varphi_{i} d m & =-\int_{\mathscr{T} \backslash E} \log \left(2(n-1) \sum_{j=1}^{n-1}\left|\psi_{n j}\right|^{2} \frac{d \mu_{j}}{d m}\right) d m+\int_{\mathscr{T} \backslash E} \log \frac{d \mu_{n}}{d m} d m \\
> & -\int_{\mathscr{T} \backslash E}\left(2(n-1) \sum_{j=1}^{n-1}\left|\psi_{n j}\right|^{2} \frac{d \mu_{j}}{d m}\right) d m+\int_{\mathscr{T} \backslash E} \log \frac{d \mu_{n}}{d m} d m>-\infty .
\end{aligned}
$$

Therefore, there is an outer function $g_{i} \in H^{2}$ such that $\left|g_{i}\right|^{2}=\varphi_{i}$.

Now let $\left\{p_{1}, \ldots, p_{n}\right\}$ be a set of $n$ polynomials and suppose $1<i<$ $n-1$. By our choice of $g_{i}$, we have

$$
\left|p_{i} g_{i}\right|^{2}<(2 n-1)^{-1}\left|p_{i} \tilde{g}_{i}\right|^{2}<(2 n-1)^{-1}\left\{\sum_{j=1}^{n-1}\left|\sum_{k=j}^{n-1} p_{k} \psi_{k j}\right|^{2} \frac{d \mu_{j}}{d m}\right\} \text { a.e. [m]. }
$$

Suppose

$$
(2 n-1)^{-1}\left\{\sum_{j=1}^{n-1}\left|\sum_{k=j}^{n-1} p_{k} \psi_{k j}\right|^{2} \frac{d \mu_{j}}{d m}\right\}>\sum_{j=1}^{n-1}\left|\sum_{k=j}^{n} p_{k} \psi_{k j}\right|^{2} \frac{d \mu_{j}}{d m}
$$

on a set $E_{0}$ of positive Lebesgue measure. We will show that on this set

$$
\left|p_{i} g_{i}\right|^{2}<\left|p_{n}\right|^{2} \frac{d \mu_{n}}{d m} \quad \text { a.e. }[m] \text {. }
$$

(The following inequalities will all hold a.e. [m] on $E_{0}$.) Indeed, by (5) for $1<j<n-1$

$$
\left|\sum_{k=j}^{n-1} p_{k} \psi_{k j}\right|^{2} \frac{d \mu_{j}}{d m}<\frac{2}{2 n-1} \sum_{i=1}^{n-1}\left|\sum_{k=i}^{n-1} p_{k} \psi_{k i}\right|^{2} \frac{d \mu_{i}}{d m}+2\left|p_{n} \psi_{n j}\right|^{2} \frac{d \mu_{j}}{d m} .
$$

(We are using here the following fact: if $|z+w|^{2} x<y$, then $|z|^{2} x<2 y+$ $2|w|^{2} x$, where $z$ and $w$ are complex and $x$ and $y$ are positive reals.) Addition of these inequalities yields

$$
\sum_{j=1}^{n-1}\left|\sum_{k=j}^{n-1} p_{k} \psi_{k j}\right|^{2} \frac{d \mu_{j}}{d m}<\frac{2(n-1)}{2 n-1}\left[\sum_{j=1}^{n-1}\left|\sum_{k=j}^{n-1} p_{k} \psi_{k j}\right|^{2} \frac{d \mu_{j}}{d m}+\sum_{j=1}^{n-1}\left|p_{n} \psi_{n j}\right|^{2} \frac{d \mu_{j}}{d m}\right] .
$$

Hence,

$$
\sum_{j=1}^{n-1}\left|\sum_{k=j}^{n-1} p_{k} \psi_{k j}\right|^{2} \frac{d \mu_{j}}{d m}<\left|p_{n}\right|^{2}\left(2(n-1) \sum_{j=1}^{n-1}\left|\psi_{n j}\right|^{2} \frac{d \mu_{j}}{d m}\right)
$$


Combining this inequality with (4) yields

$$
\left|p_{i} \tilde{g}_{i}\right|^{2}<\left|p_{n}\right|^{2}\left(2(n-1) \sum_{j=1}^{n-1}\left|\psi_{n j}\right|^{2} \frac{d \mu_{j}}{d m}\right)
$$

or

$$
\left|p_{i}\right|^{2}\left|\tilde{g}_{i}\right|^{2}\left(2(n-1) \sum_{j=1}^{n-1}\left|\psi_{n j}\right|^{2} \frac{d \mu_{j}}{d m}\right)^{-1} \frac{d \mu_{n}}{d m}<\left|p_{n}\right|^{2} \frac{d \mu_{n}}{d m}
$$

Finally, from the definition of $g_{i}$,

$$
\left|p_{i}\right|^{2}\left|g_{i}\right|^{2}<\left|p_{n}\right|^{2} \frac{d \mu_{n}}{d m} \quad \text { a.e. }[m] \text { on } E_{0}
$$

LEMMA 4.3. Let $S: H \rightarrow H$ be subnormal with standard representation ( $\mu$; $\left.F_{1}, \ldots, F_{n}\right)$, where $\mu=\left(\mu_{1}, \ldots, \mu_{n}\right)$. Suppose that, for $1<j<n$,

(a) supp $\mu_{j} \subseteq \bar{D}$,

(b) $\left.\mu_{j}\right|_{g} \ll m$,

(c) $\mu_{j}$ is not carried by the zero set of any element of $\mathrm{H}^{2}$.

Then there exists a quasiinvertible operator $Y: H^{2}\left(\mathrm{~m}^{n}\right) \rightarrow H$ such that $Y U_{\mathbf{m}^{n}}=$ $S Y$.

Proof. Let $\tilde{H}=\operatorname{TR}\left(F_{1}, \ldots, F_{n} ; W_{M}\right)$ and set $\tilde{S}=\left.W_{\mu}\right|_{\tilde{H}}$. Let $F_{k}=$ $\left(\psi_{k 1}, \ldots, \psi_{k n}\right), 1<k<n$. For convenience, we have set $\psi_{k k}=1,1<k<n$ and $\psi_{j k}=0,1<j<k<n$. Define a measure $\nu_{k}$ by

$$
d \nu_{k}=\sum_{j=1}^{n}\left|\psi_{k j}\right|^{2} d \mu_{j}
$$

For each $k, 1<k<n$, there exists a function $R_{k} \in L^{1}(m)$ such that $R_{k}>0$ a.e. $[m]$ and

$$
\int_{D}|f|^{2} d \nu_{k}<\int_{\mathscr{T}}|f|^{2} R_{k} d m<\infty
$$

for every $f \in H^{\infty}$. (Cf. [4, Lemma 4.4 $\alpha$ ] or [6, pp. 454-455]. The integral on the left-hand side is over the open disk only.) Let $g_{k}$ be an outer function in $H^{\infty}$ such that

$$
\left|g_{k}\right|^{2}=\left(1+R_{k}+\frac{d \nu_{k}}{d m}\right)^{-1}, \quad 1<k<n .
$$

Define $Y: H^{2}\left(\mathbf{m}^{n}\right) \rightarrow \tilde{H}$ by

$$
Y(0, \ldots, 0,1,0, \ldots, 0)=g_{k} F_{k} \text { and } Y U_{m^{*}}=\tilde{S} Y
$$


For arbitrary polynomials $p_{1}, \ldots, p_{n}$,

$$
\begin{aligned}
\left\|Y\left(p_{1}, \ldots, p_{n}\right)\right\|_{\mu}^{2} & =\left\|\sum_{k=1}^{n} p_{k} g_{k} F_{k}\right\|_{\mu}^{2} \\
& =\sum_{j=1}^{n} \int\left|\sum_{k=1}^{n} p_{k} g_{k} \psi_{k j}\right|^{2} d \mu_{j} \\
& <n \sum_{k=1}^{n} \int\left|p_{k}\right|^{2}\left|g_{k}\right|^{2}\left(\sum_{j=1}^{n}\left|\psi_{k j}\right|^{2} d \mu_{j}\right) \\
& <n \sum_{k=1}^{n} \int\left|p_{k}\right|^{2}\left|g_{k}\right|^{2}\left(R_{k}+\frac{d \nu_{k}}{d m}\right) d m \\
& <n\left\|\left(p_{1}, \ldots, p_{n}\right)\right\|_{m^{n}}^{2} .
\end{aligned}
$$

Thus $Y$ is bounded. By our choice of $g_{1}, \ldots, g_{n}$, their reciprocals belong to $H^{2}$; hence, $Y$ has dense range. Suppose

$$
0=Y\left(h_{1}, \ldots, h_{n}\right)=\left(\sum_{k=1}^{n} h_{k} g_{k} \psi_{k 1}, \ldots, \sum_{k=n-1}^{n} h_{k} g_{k} \psi_{k, n-1}, h_{n} g_{n}\right) .
$$

Since $\left|g_{n}\right|>0$ a.e. $\left[\mu_{n}\right]$, the function $h_{n}$ must vanish a.e. [ $\left.\mu_{n}\right]$. By hypothesis (c), we must have $h_{n}=0$ in $H^{2}$. But then the $(n-1)$ st coordinate of $Y\left(h_{1}, \ldots, h_{n}\right)$ is $h_{n-1} g_{n-1}$. By the same argument $h_{n-1}=0$. Continuing in this way, we see that $h_{1}=\cdots=h_{n}=0$; that is, $Y$ is one-to-one.

THEOREM 4.4. Suppose $S$ is subnormal. Then $S \sim \sim U_{\mathrm{m}^{n}}$ if and only if $S$ is of type $\mathcal{S}_{n}$.

Proof. If $S$ is of type $\delta_{n}$, then Lemma 4.2 and Lemma 4.3 together imply that $S \sim \sim U_{m^{n}}$. Conversely, suppose $S \sim \sim U_{m^{n}}$. Trivially, $S$ is $n$-cyclic, and by Proposition $3.5, S$ is c.n.u. Let $\left(\mu ; F_{1}, \ldots, F_{n}\right)$ be a standard representation for $S$, where $\mu=\left(\mu_{1}, \ldots, \mu_{n}\right)$. Let $H=\mathscr{T}\left(F_{1}, \ldots, F_{n} ; W_{\mu}\right)$. Without loss of generality, we may assume that $S=\left.W_{\mu}\right|_{H}$. There exist quasiinvertible operators $X: H \rightarrow H^{2}\left(\mathrm{~m}^{n}\right)$ and $Y: H^{2}\left(\mathrm{~m}^{n}\right) \rightarrow H$ such that $S \sim \sim U_{\mathbf{m}^{n}}$ via $(X, Y)$. The proof will be by induction on $n$. For $n=1$, the result is Theorem 4.5 of [4]. Suppose $n>1$ and suppose the theorem is true with $n-1$ in place of $n$.

Since $\sigma(S)=\sigma\left(U_{m}\right)=\bar{D}$, it is easy to see that supp $\mu_{k} \subset \bar{D}, 1<k<n^{\prime}$. Let $\mu_{k 0}=\left.\mu_{k}\right|_{g}$ and set $\mu_{0}=\left(\mu_{10}, \ldots, \mu_{n^{\prime} 0}\right)$. By Proposition 3.6, $\mu_{k 0} \ll m$, $1<k<n^{\prime}$. Thus, we may apply Proposition 4.1 to conclude that $n^{\prime}=n$ and that $m \ll \mu_{k 0}, 1<k<n^{\prime}$. Let $H_{0}=\mathscr{T}\left(F_{1}, \ldots, F_{n} ; W_{\mu_{0}}\right)$, and let $S_{0}=$ $\left.W_{\mu_{0}}\right|_{H_{0}}$. As in the proof of Proposition 4.1, the operator $X$ induces an operator $X_{0}: H_{0} \rightarrow H^{2}\left(\mathrm{~m}^{n}\right)$ such that $X_{0} F=X F$ for each $F \in H$ and $X_{0} S_{0}=U_{m} X_{0}$. In view of Proposition 4.1 and Lemma 4.3, $X_{0}$ has no kernel and $S_{0} \sim \sim U_{\text {r. }}$. 
To apply the inductive hypothesis, let $H_{1}=\Re\left(F_{1}, \ldots, F_{n-1} ; S_{0}\right)$, let $S_{1}=$ $\left.S_{0}\right|_{H_{1}}$, and let $X_{1}=\left.X_{0}\right|_{H_{1}}$. Then $\left.\left.U_{\operatorname{mon}}\right|_{\left(\operatorname{ran} X_{1}\right.}\right)^{-}$is an $(n-1)$-cyclic isometry with no unitary part; that is, $\left.\left.U_{\mathbf{m}^{n}}\right|_{(\operatorname{ran}} X_{1_{1}}\right)^{-} \cong U_{\mathbf{m}^{-1}}$.

Again by Proposition 4.1 and Lemma 4.3, $S_{1} \sim \sim U_{\mathbf{m}^{n-1}}$. A standard representation for $S_{1}$ is $\left(\tilde{\mu}_{0} ; F_{1}, \ldots, F_{n-1}\right)$, where $\tilde{\mu}_{0}=\left(\mu_{10}, \ldots, \mu_{n-1,0}\right)$. Hence by the inductive hypothesis, the measures $\mu_{1}, \ldots, \mu_{n-1}$ are of type $\delta$. Since quasisimilar isometries are unitarily equivalent [8], there exists a unitary operator $V_{1}: H_{1} \rightarrow H^{2}\left(\mathrm{~m}^{n-1}\right)$ such that $S_{1} \approx U_{m^{n-1}}$ via $V_{1}$. The operator $V_{1}$ extends to a unitary operator $\tilde{V}_{1}: L^{2}\left(\tilde{\mu}_{0}\right) \rightarrow L^{2}\left(\mathrm{~m}^{n-1}\right)$ such that $W_{\tilde{\mu}_{0}} \simeq W_{\mathbf{m}^{n-1}}$ via $\tilde{V}_{1}\left[5\right.$, Corollary 5.1]. Let $\varphi_{n}=\left(d \mu_{n} / d m\right)^{1 / 2}$ and define a unitary operator $V_{2}: L^{2}\left(\mu_{0}\right) \rightarrow L^{2}\left(\mathbf{m}^{n}\right)$ by $V_{2}\left(f_{1}, \ldots, f_{n}\right)=\left(\tilde{f}_{1}, \ldots, \tilde{f}_{n-1}, \varphi_{n} f_{n}\right)$, where $\left(\tilde{f}_{1}, \ldots, \tilde{f}_{n-1}\right)=\tilde{V}_{1}\left(f_{1}, \ldots, f_{n-1}\right)$. Finally, let $H_{2}=V_{2} H_{0}$, let $S_{2}=\left.W_{\mathbf{m}^{\prime}}\right|_{H_{2}}$, and set $\tilde{F}_{1}=(1,0, \ldots, 0), \quad \tilde{F}_{2}=(0,1,0, \ldots, 0), \ldots, \tilde{F}_{n-1} \stackrel{=}{=}$ $(0, \ldots, 0,1,0)$ and $\tilde{F}_{n}=V_{2} F_{n}=\left(\varphi_{1}, \ldots, \varphi_{n-1}, \varphi_{n}\right)$.

Then $H_{2}=\Re\left(\tilde{F}_{1}, \ldots, \tilde{F}_{n} ; W_{\text {men }}\right)$, and again using the fact that quasisimilar isometries are unitarily equivalent, there is a unitary operator $V_{3}: H_{2} \rightarrow$ $H^{2}\left(\mathrm{~m}^{n}\right)$ such that $S_{2} \simeq U_{\mathbf{m}^{n}}$ via $V_{3}$. Let $\left(g_{j 1}, \ldots, g_{j n}\right)=V_{3} \tilde{F}_{j}, 1<j<n$. By the unitary equivalence the following equalities hold a.e. $[\mathrm{m}]$ :

$$
\begin{array}{ll}
\sum_{k=1}^{n} g_{i k} \bar{g}_{j k}=0, & 1<i<j<n, \\
\sum_{k=1}^{n}\left|g_{j k}\right|^{2}=1, & 1<j<n, \\
\sum_{k=1}^{n} \bar{g}_{j k} g_{n k}=\varphi_{j}, & 1<j<n,
\end{array}
$$

and

$$
\sum_{k=1}^{n}\left|g_{n k}\right|^{2}=\sum_{k=1}^{n}\left|\varphi_{k}\right|^{2}
$$

For example, if $1<i<j<n$ and $p$ and $q$ are arbitrary polynomials, then

$$
\begin{aligned}
0 & =\left(p \tilde{F}_{i}, q \tilde{F}_{j}\right)_{m_{0}}=\left(p V_{3} \tilde{F}_{i}, q V_{3} \tilde{F}_{j}\right)_{m^{\prime \prime}} \\
& =\sum_{k=1}^{n} \int\left(p g_{i k}\right)\left(\overline{q g}_{j k}\right) d m=\int p \bar{q}\left(\sum_{k=1}^{n} g_{i k} \bar{g}_{j k}\right) d m .
\end{aligned}
$$

In particular, the Fourier coefficients of the function $\sum_{k=1}^{n} g_{i k} \bar{g}_{j k}$ are all zero, which is possible only if $\sum_{k=1}^{n} g_{i k} \bar{g}_{j k}=0$ a.e. $[m]$.

To finish the proof, we must show that $\int \log \left(d \mu_{n} / d m\right)>-\infty$. We assert that

$$
\frac{d \mu_{n}}{d m}=\left|\varphi_{n}\right|^{2}=\left|\operatorname{det}\left(g_{j k}\right)\right|^{2} \text { a.e. }[m] \text {. }
$$


Indeed,

$$
\begin{aligned}
& \left|\operatorname{det}\left(g_{j k}\right)\right|^{2}=\operatorname{det}\left(g_{j k}\right) \overline{\operatorname{det}\left(g_{j k}\right)}=\operatorname{det}\left(g_{j k}\right) \operatorname{det}\left(g_{j k}\right)^{*}=\operatorname{det}\left(\sum_{k=1}^{n} g_{i k} \overline{g_{j k}}\right) \\
& \begin{array}{c}
=\operatorname{det}\left(\begin{array}{ccccc}
1 & & & & \bar{\varphi}_{1} \\
& 1 & & & \bar{\varphi}_{2} \\
& & \ddots & & \vdots \\
& & & 1 & \bar{\varphi}_{n-1} \\
\varphi_{1} & \varphi_{2} & \cdots & \varphi_{n-1} & \sum_{k=1}^{n}\left|\varphi_{k}\right|^{2}
\end{array}\right] \\
=\left|\varphi_{n}\right|^{2}=\frac{d \mu_{n}}{d m} .
\end{array}
\end{aligned}
$$

But $\operatorname{det}\left(g_{j k}\right) \in H^{2 / n}$ and since $d \mu_{n} / d m$ is not the zero function,

$$
\int \log \frac{d \mu_{n}}{d m} d m>-\infty
$$

The following example shows that Theorem 4.4 does not generalize in any obvious way to include the case in which $S$ is not finitely cyclic.

ExAMPLe. Let $\mu=(m, m, \ldots)$. Let $\varphi \in L^{2}(m)$ be a cyclic vector for $W_{m}$, let $\varphi_{1}$ be the orthogonal projection of $\varphi$ into $H^{2}$, and let $\varphi_{2}=\varphi-\varphi_{1}$. Define $F_{j} \in L^{2}(\mu)$ as follows:

$$
\begin{aligned}
& F_{1}=(1,0, \ldots), \\
& F_{2}=\left(\varphi_{2}, 1,0, \ldots\right), \\
& F_{3}=(0, \bar{z}, 1,0, \ldots), \\
& F_{4}=(0,0, \bar{z}, 1,0, \ldots),
\end{aligned}
$$

Let $H=\operatorname{Tl}\left(\left\{F_{j}\right\}_{j=1}^{\infty} ; W_{\mu}\right)$ and set $S=\left.W_{\mu}\right|_{H}$. The operator $S$ is an isometry, and in view of Theorem 4.4, one might suspect that $S$ is quasisimilar to a unilateral shift of infinite multiplicity. But, this is not the case because $F=(\varphi, 0,0, \ldots) \in H$. Indeed, $\left(\varphi_{1}, 0, \ldots\right)$ is obviously in $H$. Also,

$$
\begin{aligned}
F_{2}-z F_{3} & +z^{2} F_{4}-z^{3} F_{5}+\cdots+z^{2 n} F_{2 n+2} \\
& =\left(\varphi_{2}, 0, \ldots, 0, z^{2 n}, 0, \ldots\right) \in H
\end{aligned}
$$

Therefore, $\left(\varphi_{2}, 0, \ldots\right) \in H$, and hence $F=\left(\varphi_{1}+\varphi_{2}, 0, \ldots\right) \in H$. Now $\left.S\right|_{\operatorname{Tr}(F ; S)}$ is unitary, and therefore $S$ cannot be quasisimilar to a unilateral shift.

We now come to the second main result of this section which, under appropriate conditions, extends Proposition 3.5. 
TheOREM 4.5. Let $S$ and $S^{\prime}$ be subnormal with normal decompositions

$S_{0} \oplus S_{1}: H_{0} \oplus H_{1} \rightarrow H_{0} \oplus H_{1} \quad$ and $S_{0}^{\prime} \oplus S_{1}^{\prime}: H_{0}^{\prime} \oplus H_{1}^{\prime} \rightarrow H_{0}^{\prime} \oplus H_{1}^{\prime}$. Suppose $S_{1}^{\prime}$ is of type $\delta_{n}$. Then $S \sim S^{\prime}$ if, and only if, $S_{0} \simeq S_{0}^{\prime}$ and $S_{1} \sim \sim S_{1}^{\prime}$.

Proof. If $S_{0} \simeq S_{0}^{\prime}$ and $S_{1} \sim \sim S_{1}^{\prime}$, then trivially $S \sim \sim S^{\prime}$. Conversely, suppose $S \sim \sim S^{\prime}$ via $(X, Y)$. By Proposition $3.5, S_{0} \simeq S_{0}^{\prime}$. Furthermore, by Theorem 4.4 we may assume that $S_{1}^{\prime} \cong U_{m \text { m. }}$ Let $X_{1}=\left.X\right|_{H_{1}}$ and let $P$ be the orthogonal projection of $H^{\prime}$ onto $H_{1}^{\prime}$. As shown in the proof of Proposition 3.5, $X H_{0} \subset H_{0}^{\prime}$. It follows that $\left(P X_{1}\right)\left(H_{1}\right)$ is dense in $H_{1}^{\prime}$ and that $\left(P X_{1}\right) S_{1}=$ $S_{1}^{\prime}\left(P X_{1}\right)$. Therefore, $S_{1}$ is cyclic of order at least $n$. On the other hand, repeating this argument with $Y$ in place of $X$ shows that $S_{1}$ is cyclic of order at most $n$. Thus $S_{1}$ is $n$-cyclic. By Proposition 4.1 the operator $P X_{1}$ has no kernel and the minimal normal extension of $S_{1}$ is $n$-cyclic. In view of Lemma 4.3, $S_{1} \sim \sim S_{1}^{\prime}$. Therefore the operator $S_{1}$ is of type $\delta_{n}$ by Theorem 4.4.

Corollary. Suppose $S$ is subnormal with normal decompositon $S_{0} \oplus S_{1}$. In addition, suppose $S_{1}$ is n-cyclic. Then $S$ is quasisimilar to an isometry if, and only if, $S_{0}$ is unitary and $S_{1}$ is of type $\delta_{n}$.

PROof. If $S_{0}$ is unitary and $S_{1}$ is of type $\delta_{n}$, then $S \sim \sim S_{0} \oplus U_{\text {mr. }}$. Conversely, suppose $S \sim \sim V$, where $V$ is an isometry with Wold decomposition $V_{0} \oplus V_{1}$. Then $S_{0} \approx V_{0}$ (Proposition 3.5); that is, $S_{0}$ is unitary. Furthermore, arguing as in the proof of Theorem 4.5, $V_{1}$ is a shift of finite multiplicity $n$. Therefore $S_{1} \sim \sim V_{1}$ and by Theorem $4.4 S_{1}$ is of type $\delta_{n}$.

Theorem 4.5 is false when $S_{1}^{\prime}$ is a shift of infinite multiplicity even if $S_{0}^{\prime}$ is unitary. The author is indebted to Professor Thomas Kriete for pointing out the existence of a measure with the necessary properties for this example.

EXAMPLE. Let $\mu$ be a measure such that

(1) supp $\mu \subset \bar{D}$,

(2) $\mu$ (T) $>0$,

(3) $\int \log (d \mu / d m) d m=-\infty$,

(4) $U_{\mu}$ is completely subnormal.

(Such measures exist. Indeed, let $C_{0}$ be the Cesàro operator and choose $\mu$ such that $\left(I-C_{0}\right) \approx U_{\mu}$. See Theorem 8 and the proof of Theorem 2 in [10].)

Let $\mu_{0}=\left.\mu\right|_{g}$ and let $\mu_{0}=\left(\mu_{0}, \mu_{0}, \ldots\right)$. Let $\nu=(\mu, m, m, \ldots)$ and let $\nu^{\prime}=(m, m, \ldots)$. Then $W_{\mu_{0}} \oplus U_{\nu} \sim \sim W_{\mu_{0}} \oplus U_{\nu}$, but $U_{\nu}$ and $U_{\nu}$ are not quasisimilar.

Proof. Define $X: L^{2}\left(\mu_{0}\right) \oplus H^{2}(\nu) \rightarrow L^{2}\left(\mu_{0}\right) \oplus H^{2}(\nu)$ and $Y: L^{2}\left(\mu_{0}\right) \oplus$ $H^{2}(\nu) \rightarrow L^{2}\left(\mu_{0}\right) \oplus H^{2}(\nu)$ by

$$
X\left(\left(f_{1}, f_{2}, \ldots\right) \oplus\left(g_{1}, g_{2}, \ldots\right)\right)=\left(g_{1}, f_{1}, f_{2}, \ldots\right) \oplus\left(g_{2}, g_{3}, \ldots\right)
$$


and

$$
Y\left(\left(f_{1}, f_{2}, \ldots\right) \oplus\left(g_{1}, g_{2}, \ldots\right)\right)=\left(f_{1}, f_{2}, \ldots\right) \oplus\left(\varphi g_{1}, g_{2}, \ldots\right) .
$$

Here, $\varphi$ is an outer function in $H^{\infty}$ such that the mapping $g \mapsto g \varphi$ defines a bounded, quasiinvertible operator from $H^{2}(m)$ into $H^{2}(\mu)$ (cf. Lemma 4.3). An easy verification shows that

$$
W_{\mu_{0}} \oplus U_{\nu} \sim W_{\mu_{0}} \oplus U_{\nu} \text { via }(X, Y)
$$

Now, suppose there exists a bounded operator $X_{1}: H^{2}(\nu) \rightarrow H^{2}(\nu)$ such that $X_{1} U_{v}=U_{v} X_{1}$. Let $H$ be those elements in $H^{2}(v)$ of the form

$$
(f, 0,0, \ldots), \quad f \in H^{2}(\mu) .
$$

By [10, Theorem 1] $\left.X_{1}\right|_{H}$ is the zero operator because of condition (3) on $\mu$. Therefore $U_{v}$ and $U_{v}$ cannot be quasisimilar.

\section{REFERENCES}

1. Joseph Bram, Subnormal operators, Duke Math. J. 22 (1955), 75-94.

2. Arlen Brown, $A$ version of multiplicity theory, Topics in Operator Theory, Math. Surveys, no. 13 (Carl Pearcy, Ed.), Amer. Math. Soc., Providence, R. I., 1974.

3. W. Stuart Clary, Equality of spectra of quasisimilar hyponormal operators, Proc. Amer. Math. Soc. 53 (1975), 88-90.

4. Quasisimilarity and subnormal operators, Ph. D. dissertation, Univ. of Michigan, 1973 (unpublished).

5. R. G. Douglas, On the operator equation $S^{*} X T=X$ and related topics, Acta Sci. Math. (Szeged) 30 (1969), 19-32.

6. Kenneth Hoffman and Hugo Rossi, Extensions of pasitive weak ${ }^{*}$-continuous functionals, Duke Math. J. 34 (1967), 453-466.

7. T. B. Hoover, Hyperinoariant subspaces for n-normal operators, Acta Sci. Math. (Szeged) 32 (1971), 109-119.

8. Quasisimilarity of operators, Illinois J. Math. 16 (1972), 678-686.

9. Richard V. Kadison and I. M. Singer, Three test problems in operator theory, Pacific J. Math. 7 (1957), 1101-1106.

10. Thomas L. Kriete and David Truth, On the Cesàro operator, Indiana Univ. Math. J. 24 (1974), 197-214.

11. Bernard B. Morrel, A decomposition for some operators, Indiana Univ. Math. J. 23 (1973), 497-511.

12. Joseph G. Stampfli and Bhushan L. Wadhwa, An asymmetric Putnam-Fuglede theorem for dominant operators, Indiana Univ. Math. J. 25 (1976), 359-365.

13. Bela Sz-Nagy and Ciprian Foias, Harmonic analysis of operators on Hilbert space, American Elsevier, New York, 1970.

Department of Mathematics, Fordham Universtry, BronX, New York 10458 